\title{
COLSTON RESEARCH SOCIETY
}

\section{SYMPOSIUM ON THE SUPRA-RENAL CORTEX}

This symposium, held at Bristol University from April 1 to 3, 1952, was mainly concerned with chemical and biochemical research only indirectly linked with clinical medicine, but certain items were of interest and importance to rheumatologists.

Prof. C. H. Li (California), Prof. F. G. Young (Cambridge), and DR. C. J. O. R. Morris (London) discussed methods of potentiating and fractionating ACTH and its bio-assay. The active principle seemed to be a basic polypeptide. Prof. Young thought that there were probably at least two active principles, corresponding to the adrenal weight increasing and ascorbic acid depleting fractions. It was agreed that the bio-assay by ascorbic acid depletion fixed by WHO did not correlate with other methods, such as adrenal weight, thymus inhibition, cholesterol depletion, whitecell reduction in the thoracic duct, or eosinopenic responses. In the discussion Dr. E. B. Astwood (Boston, Mass.) pointed out that unhypophysectomized man might not react like a hypophysectomized animal. Crude ACTH contained in addition to pure corticotrophin, growth hormone, a fat-mobilizing principle and intermedin, the melanophore stimulating factor.

Prof. J. M. Yoffey (Bristol) discussed the histology of the adrenal cortex and the evidence for the action of ACTH on the zona reticularis.

Prof. F. Verzar (Basel) appealed for the interrelationing and unification of the cortical hormones. He drew attention to the production of oxysteroids on perfusion of the adrenal with DOCA, and to the fact that this hormone can maintain secondary sex functions in adrenalectomized animals. $\mathrm{He}$ also stated that small repeated doses of gluco-corticoids had the electrolytic effects associated with DOCA. Gluco-corticoids affected the mobilization of glucose in the cell, from glycogen, fat, or protein, with subsequent deposition of glycogen. The potassium changes were secondary to these changes. Dr. H. Hoagland (Shrewsbury, Mass.) had isolated eight adrenal steroids after ACTH administration. Corticosterone and 17-hydroxy corticosterone made up the bulk, but about 3 per cent. cortisone and 2 per cent. desoxycorticosterone were also obtained. It was agreed that a considerable interconvertibility of the adrenal cortical hormones existed, but many who took part in the discussion were not prepared to believe in the complete overlap of gluco- and mineralocorticoids and sex hormones suggested by Prof. Verzar; it was asked, for instance, why a small dose of cortisone was so essential to the satisfactory control of a difficult case of Addison's disease.

DR. M. Vogt (Edinburgh) discussed the mechanism of stimulation of the anterior pituitary by adrenaline, stress reduction of corticoids, and stimulation of the hypophysis, and also the mechanism of direct stimulation of the adrenal cortex.

Prof. S. Zuckerman (Birmingham) drew attention to the need for biological rather than chemical criteria in endocrinology, and to the danger of disarticulation of minutiae, as their interaction was so complicated. For instance, he suggested that both cortisone and sex hormones could be produced by the adrenal cortex and gonads on stimulation either by ACTH or by gonado-trophin. Dr. S. J. Folley (Reading), discussing lactation, mentioned work done with DOCA and cortisone implants. He thought one of the fundamental effects of the gluco-corticoids was on glucogenesis from the fatty acids. Dr. F. T. G. Prunty (St. Thomas's Hospital, London) also emphasized the need to integrate chemical and biochemical observations; he illustrated his point by quoting such complicating factors as the synergic effects of luteinizing hormone and cortisone, and the apparent difficulty in interpreting ACTH potency by ascorbic acid depletion when scorbutic guinea-pigs react very well to ACTH.

Prof. G. F. Marrian (Edinburgh) summarized his present work. Formaldehydogenic steroid is lost on allowing acid urine to stand and in extraction at $p \mathrm{H} \mathrm{I}$., glucuronidase hydrolysis increased the yield seven- or eight-fold. The method was not yet, however, properly standardized, and the hydrolysis did not affect a conjugated fraction, and was not yet, therefore, a method suitable for routine application.

Dr. H. J. Robinson (from Messrs. Merck Ltd., U.S.A.) showed that with large doses of cortisone or ACTH (5 mg. daily) in rabbits, pneumococcal lesions were increased in size, and the leucocyte reaction in the tissues, ingestion of bacteria, and immunity titre were all delayed with increase in mortality. With small doses $(0.5 \mathrm{mg}$. daily or less), however, the treated group reacted better than the controls. Cortisone had little effect on toxins, but did not reduce the lethal dose of pyrogens. Dr. R. R. H. Lovell (London) said that in man the dose of cortisone necessary to reduce experimental skin inflammations was generally higher than that found effective therapeutically. Perhaps this was why dissemination of infection occurred less commonly than might be expected from animal experiments. Dr. M. Reiss (Bristol) drew attention to the importance of distinguishing the effects of small and large doses of these hormones, stating that many years ago he had found increased ingestion of carmine by the reticulo-endothelial system during treatment with small doses of crude ACTH - the opposite effect to that obtained with present-day large doses. 
Dr. H. Hoagland (Shrewsbury, Mass.), and Drs R. E. Hemphill and M. Reiss (Bristol) then discussed the effects and abnormalities of hormones in mental disease. Among other matters, the high and irregular ketosteroid excretion, poor reaction to stress, and clinical deterioration with hyper-adrenalism in schizophrenia was agreed; whether these results occurred post hoc or propter hoc was uncertain, but in the United States adrenalectomy had been tried as a method of treatment. In psychosis, E.C.T., insulin, testosterone, and ACTH treatment often had considerable value, but their effect was complicated. Hyperthyroidism was a brake on the reaction of ACTH.

Prof. G. R. Cameron (University College Hospital, London) described possible changes in the tissues resulting from the use of ACTH and cortisone, and the inhibition of the hyaluronidase spreading effect, the histamine and leucotactic polypeptide reaction, and the diffusion of fluorescein from the blood vessel of the eye. Delayed healing was also demonstrated, but it was the speed and amount of repair that was altered and not the general pattern. The question of inhibition of cell mitosis is still uncertain.

Dr. P. L. KroHN (Birmingham) then discussed the effect of cortisone in controlling the inflammatory reaction which prevents homogeneous skin grafts from taking; again the reaction is delayed not altered, the grafts eventually succumbing. Dr. O. A. Trowell (Harwell) showed a delayed toxic effect of cortisone on lymphocytes in vitro; there was no effect on macrophages. Prof. J. M. Yoffey demonstrated an absolute increase in erythroid and myeloid cells in the bone marrow with ACTH; there was little if any change in the absolute number of lymphocytes in the marrow. Dr. K. Aterman described experiments suggesting an effect of cortisone on wellformed fibrous tissue, but this was challenged by other workers.

Dr. DWight J. INGLe (from the Upjohn Coy., U.S.A.) discussed the synergic effect of oestrogens and cortisone in the production of diabetes and control of hair growth in rats. With regard to "stress" and "diseases of adaption" he felt that stimulation of the adrenals was a defence mechanism which did not constitute hyper-corticism, but might contribute to the symptomatology of disease without actually causing it.

Prof. H. Heller (Bristol) outlined the influence of the adrenal cortex on mineral and water metabolism.

Mr. L. R. BRoster (Charing Cross Hospital, London) described clinical varieties of virilism and the correlation between the degree of virilism and the degree of fuchsin staining of the adrenal. Dr. Jolley emphasized the value of cortisone in treating congenital adrenal hyper plasia, especially if the treatment was started early? Dr. Hoagland mentioned the very satisfactory results? of total adrenalectomy +orchidectomy in carcinoma of: the breast and prostate.

DR. E. B. Astwood (Boston, Mass.) described the action of cortisone and ACTH as anti-allergic or anti phlogistic. Hydro-cortisone was said to be slightly moreळ active than cortisone both clinically and in its " electro lytic " effects, and was best used locally or orally, sinces intramuscular injections were absorbed very slowly $\vec{\circ}$ Local treatment could be used for the eye, for joints;and as an aerosol for asthma. A much purified prepara $\vec{w}$ tion of ACTH had been produced which used intramuscularly was much more potent clinically than was suggested by assay or might be expected from its con-centration. Intravenously, however, this enhanced potency was much less spectacular. Considerable progress had been made in delaying the absorption of ACTH by administering it in an oil or gelatin base.

DR. G. D. KersLey (Bath) urged that each patient should be considered on his own merits, remembering $ᄃ$ the temporary blanketing effect on allergy and fibrosis $\widetilde{\nabla}$ These principles must be applied after considerationof the patient's history, the stage of the disease, 0 other complications, and the social background, since $N$ both hyperergy and scarring could be either helpful or harmful. In rheumatic fever the value of these hormones? was doubtful, in spondylitis they vied with radiotherapy in the treatment of young women only, and in rheumatoido arthritis they were very helpful in the short-term treat $-\frac{0}{D}$ ment of acute attacks due to a temporary cause, to cover@ rehabilitation, in manipulation, and sometimes with $\overrightarrow{0}$ surgery. He then described two cases of rheumatoid 3 arthritis clinically resistant to $400 \mathrm{mg}$. ACTH daily and? $100 \mathrm{mg}$. intravenously, and one who failed to react to $500 \mathrm{mg}$. intramuscular cortisone daily or $50 \mathrm{mg}$. intraarticular hydro-cortisone. Finally, he pleaded for $a_{0}^{\circ}$ balanced view of the value of cortisone. In the discussion, Dr. P. Ellman (London) described a case?. of Addison's disease with rheumatoid arthritis wellin controlled with $1.5 \mathrm{mg}$. DOCA and $25 \mathrm{mg}$. cortisone daily. Dr. West mentioned a patient with a duodenal ulcer which healed while he was receiving cortisone, and? another patient who developed severe amyloid disease. $\rightarrow$ Dr. J. J. R. Duthie (Edinburgh) drew attention to the absence of any correlation between the severity of then disease and the therapeutic dosage of cortisone. $\mathrm{He}^{\circ}$ also said the results of cortisone and intravenous iron N therapy combined in rheumatoid arthritis were goodN in that the red-cell count increased. 